\title{
Factibilidad de análisis biomolecular en base a la metilación del promotor de 06-metilguanina-ADN metiltransferasa mediante técnica de PCR anidado en glioblastoma multiforme en el Hospital Carlos Van Buren, Chile Feasibility of biomolecular analysis based on methylation of O6-methylguanine-DNA methyltransferase promoter by nested PCR technique in glioblastoma multiforme at Carlos Van Buren Hospital, Chile
}

\author{
Esteban F. Espinoza-García ${ }^{1}$, Carolina Rejas-Gallegos², Andrés Horlacher-Kunstmann¹, Francisco M. González-Vicuña \\ Mario Párraga-San Román² \\ 1 Servicio de Neurocirugía, Hospital Carlos Van Buren, Valparaíso, Chile. \\ 2 Centro de Investigaciones Biomédicas, Universidad de Valparaíso, Chile.
}

Rev. Chil. Neurocirugía 45: 200-208, 2019

\section{RESUMEN}

El glioblastoma multiforme es el subtipo de gliomas más frecuente en adultos, con una pobre sobrevida promedio posterior al diagnóstico. Sin terapia, la sobrevida de los pacientes posterior al diagnóstico no excede los 3 meses, incrementándose a un promedio de 12 a 15 meses si se aplica el tratamiento óptimo, que incluye resección quirúrgica, radioterapia y quimioterapia con temozolomida. El objetivo primario de este estudio es determinar la factibilidad de realizar el análisis biomolecular de muestras histológicas con diagnóstico de Glioblastoma Multiforme en el Hospital Carlos Van Buren utilizando PCR anidado para determinar metilación del promotor de MGMT, en el período comprendido entre 2010 y 2014. Como objetivo secundario, se pretende evaluar la correlación entre el tiempo de sobrevida y el estado de metilación el promotor de MGMT. Se estudió el estado de metilación de MGMT en 12, logrando un 57,1\% de éxito en la amplificación. Un 83,3\% resultó no metilado, con una sobrevida de 9,2 meses, comparado con 21 meses de sobrevida del grupo metilado $(p=0,15)$. Como conclusión, la técnica de PCR anidado es factible de realizar en nuestro centro, requiriéndose estudios prospectivos para evaluar el valor predictivo, aunque se observó una tendencia a que la metilación del promotor de MGMT se relaciona con aumento en el tiempo de sobrevida posterior al diagnóstico.

Palabras clave: Glioblastoma, Reacción de Polimerasa en Cadena, Sobrevida.

\begin{abstract}
Glioblastoma multiforme is the most frequent subtype of gliomas in adults, with a poor survival rate after diagnosis. Without treatment, the mean survival time doesn't exceed 3 months after diagnosis, increasing up to 12 to 15 months if the optimal treatment is given, including surgical resection, radiotherapy and chemotherapy using temozolomide. The main objective of this study is to evaluate the feasibility to do a biomolecular analysis of histological samples at Carlos Van Buren Hospital using nested PCR technique to determine methylation of MGMT promoter between 2010 and 2014. As an additional objective, we pretended to evaluate the correlation between mean survival time and MGMT promoter methylation status. The MGMT methylation status was studied in twelve samples, achieving a $57,1 \%$ amplification success. An $83,3 \%$ was found in a non-methyl-
\end{abstract}


ated status, with 9.2 months of mean survival time, in contrast to 21 months in the methylated group $(p=0.15)$. We concluded that nested PCR technique is feasible to do in our center, requiring prospective studies to evaluate a predictive value, although an increased mean survival time after diagnosis and methylated status was related.

Key words: Glioblastoma, Polymerase Chain Reaction, Survival.

\section{Introducción}

El término "tumor cerebral" engloba a un conjunto de neoplasias, cada una con su propia biología, pronóstico y tratamiento. Estos tumores se identifican mejor con el término "neoplasias intracraneales", ya que no todas emergen del tejido cerebral ${ }^{1}$.

Alrededor de un $80 \%$ de los tumores cerebrales malignos primarios corresponden a gliomas ${ }^{2}$. El término "glioma" comprende a todos los tumores que tienen un origen glial, los cuales incluye tumores astrocíticos, oligodendrogliomas, ependimomas y gliomas mixtos ${ }^{2,3}$, con una incidencia general de gliomas de 4,67 a 5,73 por 100.000 personas $^{4,5}$. La Organización Mundial de la Salud los clasifica según la diferenciación celular en 4 grupos, constituyendo el grado I de la OMS tumores diferenciados y con comportamiento menos agresivo, hasta el grado IV que no son diferenciados y que presentan el mayor comportamiento agresivo ${ }^{6}$. El subtipo más común y más letal es el glioblastoma multiforme (GBM), grado IV de la OMS, cuya incidencia se describe entre 0,59 a 3,69 por 100.000 personas, siendo el tercer tipo tumoral más frecuentemente reportado, después de los meningiomas $(36,6 \%)$ y los tumores pituitarios $(15,9 \%)^{5,7-11}$. A partir del año 2016 , la OMS los clasifica según mutaciones en gen IDH en IDH-wildtype e IDH-mutant. Los primeros comprenden al $90 \%$ de los casos, correspondiendo a los definidos clínicamente como primarios o "de novo", mientras que los del subtipo IDH-mutant corresponden al $10 \%$ de los casos, relacionados con los glioblastomas denominados secundarios, en los que se pesquisa previamente un glioma de menor grado que presenta progresión a glioblastoma, siendo estos últimos preferentemente de la población más joven ${ }^{12}$.

Esta enfermedad afecta comúnmente a la población adulta, siendo el rango de edad más frecuentemente afectado entre 45 a 70 años, con ligera predominancia masculina ${ }^{13}$. La mayoría de los casos son de aparición esporádica, aunque se ha descrito una mayor incidencia de GBM en pacientes con antecedentes de exposición a radiación ionizante e historia de síndromes neurocutáneos, como la esclerosis tuberosa y la neurofibromatosis ${ }^{2,14,15}$. Este tipo tumoral se puede desarrollar a partir de un glioma de menor grado, como un glioma de bajo grado (grado II de la OMS) o de un astrocitoma anaplásico (grado III de la OMS), sin embargo, la mayoría se desarrolla de novo sin evidencia de una lesión preexistente ${ }^{6}$. Las manifestaciones clínicas, como cualquier lesión neurológica, depende de la localización anatómica y la extensión de la invasión tumoral. Según el Glioma Outcomes Project, un 53\% a $57 \%$ de los pacientes la sintomatología inicial fue cefalea, mientras que un $56 \%$ de los gliomas grado III y $23 \%$ de los gliomas grado IV se asociaron a crisis convulsivas. Otros síntomas comúnmente vistos al diagnóstico incluyeron cambios cognitivos, déficit visual, paresia y trastornos del lenguaje ${ }^{16}$.

Las modalidades actuales de tratamiento incluyen a la cirugía, radioterapia y quimioterapia con temozolomida. Dada la agresividad de esta neoplasia, la mejor combinación de tratamientos sólo puede aumentar la sobrevida del paciente a un período de 12 a 15 meses posterior al diagnóstico, en promedio $^{17}$.

\section{Cirugía en el tratamiento del glioblastoma multiforme}

Los nuevos avances en el campo de imagenología, como la neuronavegación, o avances como la resección guiada con fluorescencia han permitido aumentar la seguridad en el manejo quirúrgico y han permitido un incremento en el porcentaje de exéresis, lo que ha significado casi doblar las tasas de sobrevida desde 3 a 4 meses hasta 7 a 12 meses, aunque relacionados principalmente por la mayor resección de tejido tumoral que permiten estas herramientas complementarias ${ }^{18,19}$

La resección extensa tumoral en glioblastomas es un desafío quirúrgico, ya que estos tumores son invasivos, infiltrantes y frecuentemente involucran áreas elocuentes ${ }^{20}$. Se ha planteado la hipótesis de que un mayor porcentaje de exéresis se relacionaría con un incremento en el pronóstico del paciente, disminuyendo la masa tumoral y facilitando la respuesta a la terapia adyuvante $^{16,21-23}$. Se ha visto mayor beneficio en pacientes con resección completa o resección cercana a completa, definida esta última como aquella que, en el control imagenológico postoperatorio con resonancia magnética no presenta realce de contraste, independiente de alteración en secuencias largas. EI beneficio disminuye al realizar una resección subtotal, definida como aquella que en la resonancia magnética postoperatoria presente realce con contraste nodular residual ${ }^{24}$.

\section{Radioterapia adyuvante}

La radioterapia fraccionada de haz externo es el pilar de la terapia adyuvante en astrocitomas de alto grado. Estudios aleatorizados de las décadas del 70 y 80 conducidos por el Brain Tumor Study Group demostraron que la radioterapia postoperatoria aumentaba la sobrevida de manera significativa estadísticamente en comparación con sólo la cirugía. En uno de estos ensayos, Walker et $\mathrm{al}^{19}$ aleatorizaron a 303 pacientes en cuatro grupos, resultando en un aumento de la sobrevida estadísticamente significativa en todos los grupos que recibieron radioterapia. Los tiempos de sobrevida promedio fueron de 4,3 meses para el grupo con terapia de soporte, 6,3 meses para el grupo que sólo recibió quimioterapia, 9,4 meses para el grupo con sólo con radioterapia y de 10,1 meses para el grupo con quimioterapia y radioterapia combinada. En una revisión sistemática realizada por Laperriere et al se confirmó este beneficio ${ }^{25}$. 
Con respecto a la extensión de la radioterapia, no se demostró beneficio de la radioterapia en todo el cerebro comparada con campos más restrictivos ${ }^{25,26}$. La evidencia con respecto a la historia natural ha demostrado una tendencia a la recurrencia local en un área de 1 $\mathrm{cm}$ a $2 \mathrm{~cm}$ de la lesión inicial. La práctica clínica actual considera radiación externa focalizada en el área de lesión primaria y $2 \mathrm{~cm}$ de márgenes ${ }^{19,21,24}$

\section{Quimioterapia con temozolomida}

La temozolomida corresponde a una clase de agentes alquilantes conocidos como imidazotetrazinas, con una estructura similar a la dacarbazina, con un anillo imidazol ${ }^{22}$. Fue sintetizada por Stevens y colaboradores en $1984^{23}$, demostrando su actividad antineoplási$\mathrm{ca}^{23,25}$. La penetración de la temozolomida en el sistema nervioso central fue estudiada en ratas y monos Rhesus, revelando que los niveles del fármaco en el cerebro y en el líquido cefalorraquídeo son aproximadamente $30 \%$ a $40 \%$ de la concentración plasmática ${ }^{27}$.

El mecanismo de acción consiste en que el ion metildiazonio (MTIC), compuesto activo, metila los residuos guanina en el ADN, resultando en la formación de $\mathrm{O} 6$ y N7-metil-guanina. La formación de 06-metilguanina es primariamente responsable de los efectos citotóxicos de la temozolomida. Cuando el grupo metilo no logra ser retirado previo a la división celular, se desencadena la reparación a través de la vía de error en la unión de bases, resultando ser altamente citotóxico, ya que se produce roturas de doble cadena del ADN y con esto se activan las vías de apoptosis ${ }^{27}$.

Un importante estudio en relación con la terapia adyuvante en GBM fue publicado en 2005 por Stupp et al ${ }^{17}$. En este estudio se logró demostrar con nivel I de evidencia que la utilización de temozolomida como quimioterapia adyuvante en el manejo de GBM aumentaba la sobrevida de forma significativa estadísticamente. Un total de 573 pacientes fueron aleatorizados a recibir sólo radioterapia (60 Gy administrados 5 días por semana por 6 semanas) o radiación en combinación con temozolomida (en dosis de $75 \mathrm{mg} / \mathrm{m}^{2}$ de superficie corporal, 7 días a la semana concurrentemente con la radioterapia y 6 ciclos de 150$200 \mathrm{mg} / \mathrm{m}^{2}$ posterior a la radioterapia).
Los grupos fueron repartidos en grupos comparables según edad, extensión de resección tumoral y estado funcional. Los resultados demostraron que hubo un incremento significativo en el brazo de temozolomida en cuanto a sobrevida libre de progresión (6,9 meses vs 5 meses) y sobrevida promedio (14,6 meses vs 12,1 meses) y un incremento sustantivo en la sobrevida a los 2 años $(27,2 \%$ vs $10,9 \%)$ y a los 5 años $(9,8 \%$ vs $1,9 \%)$

\section{Rol de la enzima 06-metilguanina- ADN metiltransferasa (MGMT) en la resistencia a los agentes alquilantes}

La MGMT es una enzima de reparación de $A D N$, que remueve rápidamente el grupo metilo (alquilante) de la posición $\mathrm{O} 6$ de la guanina, transfiriéndolo a una cisteína de su propia estructura, quedando irreversiblemente inactiva. El nucleótido afectado por el agente alquilante recupera su forma original, evitándose los efectos citotóxicos de los agentes como la temozolomida e impidiendo la activación de la apoptosis celular. Los niveles altos de MGMT activa en células tumorales resulta en una resistencia a agentes alquilantes, mientras que la depleción de la proteína puede estar asociada a mejor evolución ${ }^{28,29}$. De esta manera, si se inhibe la actividad del MGMT mediante la metilación del promotor del gen, resultaría en una disminución en la inactivación de los agentes utilizados en la quimioterapia, por lo que esta modificación le otorga a un paciente un mejor pronóstico.

Con respecto a los estudios clínicos, se realizó un estudio multicéntrico en Alemania y Suiza que estudió la metilación el promotor de MGMT y su relación con el pronóstico, denominado NOA-08. En este estudio multicéntrico se aleatorizaron 412 pacientes con diagnóstico de GBM o astrocitoma anaplásico, mayores de 65 años, a recibir terapia complementaria con sólo temozolomida o sólo radioterapia, observándose que en aquellos pacientes que se encontró metilación del promotor de MGMT se asoció de manera estadísticamente significativa con una mayor sobrevida (11,9 meses vs 8,2 meses) y mayor sobrevida libre de evento $(5,7$ meses vs 3,5 meses), definida esta última como el tiempo entre el diagnóstico y la primera progresión de la enfermedad o entre el diagnóstico y fallecimiento (dependiendo si existió progresión de la enfermedad dentro de la evolución). Se observó además que, en el grupo con metilación del promotor, hubo con un aumento del período libre de evento en el grupo tratado con temozolomida $(8,4$ meses vs 3,3 meses), sin observarse esta diferencia en el grupo tratado con radioterapia ${ }^{30}$.

Otro estudio multicéntrico que evidenció este beneficio fue el Nordic Trial. En este estudio se aleatorizaron 342 pacientes con diagnóstico de novo de Glioblastoma multiforme grado IV OMS, mayores de 60 años, a recibir temozolomida, radioterapia hipofraccionada o radioterapia estándar, destacando que en el grupo de pacientes en los que se encontró metilación del promotor MGMT se evidenció un aumento en la sobrevida en el grupo tratado con temozolomida, en comparación con los pacientes sin metilación del promotor (9,7 meses vs 6,8 meses). En relación con el estado de metilación, no se observó diferencias estadísticamente significativas en los grupos tratados con radioterapia ${ }^{31}$

En el estudio realizado por Stupp en 2009, conducido por la European Organisation for Research and Treatment of Cancer (EORTC) y National Cancer Institute of Canada Clinical Trials Group (NCIC), se demostró también que la metilación del promotor del MGMT fue un factor predictor de buen pronóstico, especialmente en el grupo tratado con temozolomida más radioterapia ${ }^{32}$.

\section{Técnica de PCR anidado para estudio de metilación del promotor de MGMT en el Hospital Carlos Van Buren}

El Hospital Carlos Van Buren es un hospital de alta complejidad, ubicado en la ciudad de Valparaíso, el cual concentra la patología neuroquirúrgica compleja de 3 regiones de Chile. Existe un estudio previo realizado en este centro en conjunto con la Universidad de Valparaíso, como proyecto de tesis de especialidad médica de Dr. Felipe Rey, el cual evaluó el perfil molecular del glioblastoma multiforme mediante la determinación de metilación del promotor de MGMT por PCR anidado entre 2004 y 2009, estudiándose 19 muestras tumorales, donde el $68 \%$ presentaba metilación del promotor MGMT 
obtenido mediante estudio con la técnica de PCR anidado, con una mediana de sobrevida de 11,1 meses en el grupo metilado versus 5,5 meses en el grupo no metilado $p=0,1$ ). Además, se evidenció que el porcentaje de sobrevida a 1 año y 2 años de los pacientes con MGMT metilado era de $45 \%$ y $30 \%$ respectivamente, en comparación con la sobrevida al año y a dos años en el grupo no metilado, que correspondió a $16 \%$ y $0 \%$ respectivamente. Todo lo anterior, utilizando técnica de PCR anidado para determinación de metilación del promotor de $\mathrm{MGMT}^{33}$. Existe un estudio publicado en 2008 en Surgery Neurology, en donde se establecen los factores clínicos, imagenológicos y de tipo histológico que determinan el pronóstico en los pacientes con gliomas de alto grado en Chile, siendo evaluados 103 pacientes con diagnóstico de glioma de alto grado, estudiándose como factores la edad, histología y Karnofsky, determinándose valores estadísticamente significativos para aquellos ítems ${ }^{34}$. El estudio de factores pronósticos en tumores cerebrales se ha convertido en una importante área de estudio a nivel internacional, por ello, se realizó un estudio del estado de metilación del promotor de MGMT en pacientes operados entre 2010 y 2014 para evaluar su aporte en el ámbito clínico.

El objetivo del presente trabajo es evaluar la factibilidad de realizar el estudio biomolecular en el Hospital Carlos Van Buren, en conjunto con la Universidad de Valparaíso, utilizando la técnica de PCR anidado para determinar el estado de metilación del promotor del MGMT.

\section{Material y Método}

Se realizó un estudio analítico de cohorte retrospectivo, estudiándose a los pacientes operados con diagnóstico de glioblastoma multiforme OMS IV en el Hospital Carlos Van Buren entre los años 2010 y 2014. Los siguientes fueron los criterios de inclusión: paciente operado en el Hospital Carlos Van Buren entre los años 2010 y 2014; diagnóstico histológico de glioblastoma multiforme OMS IV; edad entre 18 y 70 años, esto para que los resultados fueran comparables con resultados internacionales; muestra tumoral disponible en Servicio de Anatomía Patológica del Hospital. Se excluyeron pacientes con biopsia distinta a glioblastoma multiforme, aquellos que presentaran muestra tumoral insuficiente o de mala calidad, con cantidad insuficiente de ADN para realizar estudio de metilación de promotor de MGMT.

\section{Forma de reclutamiento y aspectos bioéticos}

Se utilizo los registros de Anatomía Patológica para identificar las muestras de pacientes con diagnostico de glioblastoma multiforme OMS IV y realizar el estudio de sobrevida posterior. Dentro del protocolo de este estudio, se dejo consignado que a los pacientes vivos se le solicitara autorización al paciente o a sus familiares mediante contacto telefónico, realizado por el médico tratante, y posterior firma de consentimiento informado. Se solicito autorización a la Dirección del Hospital Carlos Van Buren para poder realizar los estudios en muestras de pacientes fallecidos. Este estudio fue evaluado por el Comité de Bioética de la Facultad de Medicina de la Universidad de Valparaíso y por el Comité Ético-Científico del Servicio de Salud Valparaíso-San Antonio, siendo aprobado por ambos comités. Las muestras de tejido tumoral fueron extraídas del Servicio de Anatomía Patológica para su posterior estudio biomolecular.

\section{Análisis molecular}

De los pacientes con diagnóstico de Glioblastoma Multiforme, se seleccionó en la lámina de diagnóstico una región con mayor cantidad de células tumorales, escogidas por dos anatomopatólogos del Hospital Carlos Van Buren. Tomando como referencia esta región, se extrajo de las muestras parafinadas un segmento tumoral de aproximadamente $2 \mathrm{~mm}^{3}$, necesario para realizar la extracción de ADN. Una vez obtenidos los segmentos tumorales, las siguientes etapas fueron realizadas en el Centro de Investigaciones Biomédicas de la Universidad de Valparaíso.

Posteriormente, se procedió a realizar la extracción de ADN utilizando el Kit EZNA Tissue DNA (Omega Bio-Tek), según instrucciones del fabricante. Una vez obtenido el ADN, se procedió a cuantificar las concentraciones de ADN mediante espectrofotometría
UV a $260 \mathrm{~nm}$, con la relación: una unidad de $A_{260 \mathrm{~nm}}$ equivale a $50 \mathrm{ng} / \mu \mathrm{L}$ de ADNds. Para corroborar la calidad del ADN extraído, se amplificó una región del exón 2 de KRAS con oligonucleótidos específicos (FOR: 5'-GAA TGG TCC TGCA CCA GTA A-3' y REV: 5'GTG TGA CAT GTT CTA ATA TAG $\left.\mathrm{T}-\mathrm{3}^{\prime}\right)$, eligiéndose este gen por tratarse de un segmento génico no relacionado con MGMT. La preparación para realizar la reacción de PCR fue la siguiente:

- Buffer 5x: $5 \mu \mathrm{L}$

- dNTP $10 \mathrm{mM}: 0,5 \mu \mathrm{L}$

- $\mathrm{MgCl}_{2} 25 \mathrm{mM}: 1,5 \mu \mathrm{L}$

- Primer FOR KRAS: $1 \mu \mathrm{L}$

- Primer REV KRAS: $1 \mu \mathrm{L}$

- Taq Pol: 0,1 $\mu \mathrm{L}$

- Agua: $13,9 \mu \mathrm{L}$

- DNA: $2 \mu \mathrm{L}(500 \mathrm{ng}$ de ADN)

A su vez, se realizó un control positivo agregando ADN extraído de linfocitos humanos normales y un control negativo reemplazando el ADN por agua.

Se utilizó el termociclador (iCycler, BioRad) con el siguiente programa: un ciclo de desnaturalización a 95ํ $\mathrm{C}$ por 7 minutos; posteriormente la amplificación se realizó en un paso de desnaturalización a 95으 $\mathrm{C}$ por 30 segundos, un alineamiento a $52^{\circ} \mathrm{C}$ por 30 segundos y un último de extensión a $72^{\circ} \mathrm{C}$ por 30 segundos, repitiéndose esta secuencia 35 veces. Finalmente se realizó un ciclo de extensión a $72^{\circ} \mathrm{C}$ por 7 minutos y mantención a $4^{\circ} \mathrm{C}$.

Se obtuvo un fragmento de 208 bp que fue visualizado en transiluminador UV (Vilbert Lourmat) tras electroforesis en gel de agarosa al $2 \%$. El programa de electroforesis fue: $70 \mathrm{~V}, 400 \mathrm{~mA}$, por un tiempo de 45 minutos. Las muestras que no amplificaron este fragmento fueron descartadas (Figuras 1 y 2).

Posteriormente, se utilizó el kit EZ DNA Methylation-Gold (Zymo Research) segín indicación del fabricante, con el fin de convertir las citosinas no metiladas en uracilos, dejando intactas las citosinas metiladas. Se realizó una elución de $10 \mu \mathrm{L}$.

Para realizar el estudio de metilación del promotor se utilizó la técnica de PCR anidado, variante de PCR convencional en la cual se utilizan dos parejas de oligonucleótidos distintas, con la finalidad de aumentar la sensibilidad de la detección. En un primer tiempo se amplifica una zona extensa de ADN, la cual será utilizada como molde en una segunda reacción de PCR con oligonu- 


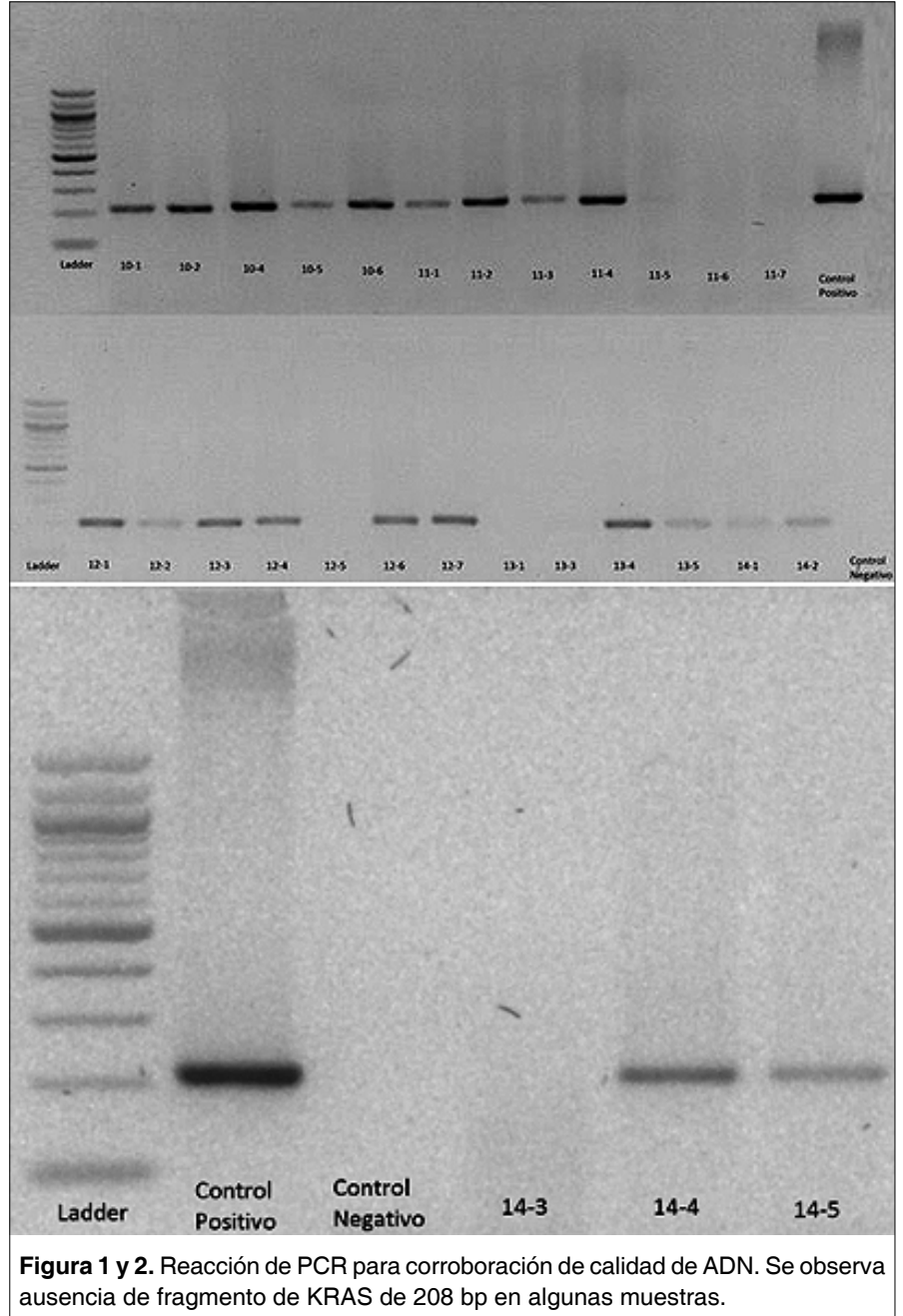

cleótidos internos ${ }^{33}$, como se muestra en la Figura 3. Para la realización de esta técnica en el presente estudio, se realizó una primera reacción de PCR con primer específico de promotor de MGMT metilado y sin metilar (FOR: 5'GGATATGTTGGGATAGTT-3' y REV: 5'-CCAAAAACCCCAAACCC-3'), con la siguiente preparación:

- Buffer 5x: $5 \mu \mathrm{L}$

- dNTP $10 \mathrm{mM}: 0,5 \mu \mathrm{L}$

- $\mathrm{MgCl}_{2} 25 \mathrm{mM}: 1,5 \mu \mathrm{L}$

- Primer FOR MGMT: $1 \mu \mathrm{L}$

- Primer REV MGMT: $1 \mu \mathrm{L}$

- Taq Pol: 0,1 $\mu \mathrm{L}$

- Agua: 7,9 $\mu \mathrm{L}$

- Producto de kit EZ DNA: Methylation-Gold: $8 \mu \mathrm{L}$

Se utilizó el termociclador con el siguiente programa:

1 ciclo de desnaturalización: 95ㅇ C por 4 minutos. do:

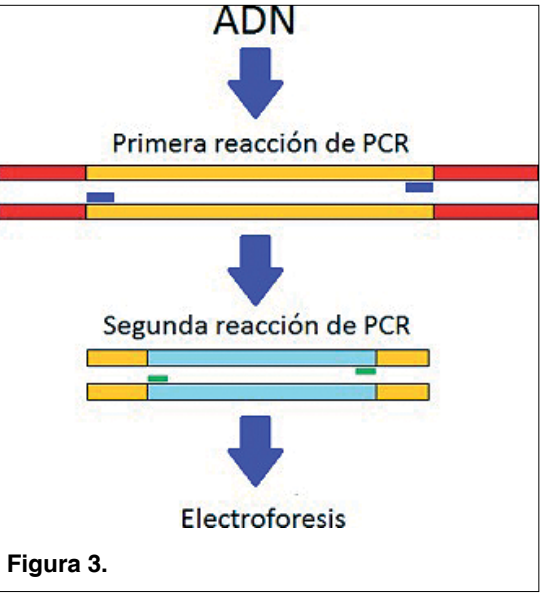

Buffer 5x: $5 \mu \mathrm{L}$

- dNTP $10 \mathrm{mM}: 0,5 \mu \mathrm{L}$

- $\mathrm{MgCl}_{2} 25 \mathrm{mM}: 1,5 \mu \mathrm{L}$

Primer FOR MGMT(M): $1 \mu \mathrm{L}$

Primer REV MGMT(M): $1 \mu \mathrm{L}$

- Taq Pol: 0,1 $\mu \mathrm{L}$

- Agua: 11,9 $\mu \mathrm{L}$

- Producto de PCR de primera reacción: $4 \mu \mathrm{L}$

Preparado para evaluar MGMT no metilado:

- Buffer 5x: $5 \mu \mathrm{L}$

- dNTP $10 \mathrm{mM}: 0,5 \mu \mathrm{L}$

- $\mathrm{MgCl}_{2} 25 \mathrm{mM}: 1,5 \mu \mathrm{L}$

- Primer FOR MGMT(U): $1 \mu \mathrm{L}$

- Primer REV MGMT(U): $1 \mu \mathrm{L}$

- Taq Pol: 0,1 $\mu \mathrm{L}$

Agua: $11,9 \mu \mathrm{L}$

- Producto de PCR de primera reacción: $4 \mu \mathrm{L}$

Se utilizó el termociclador con el siguiente programa:

- 5 ciclos de preamplificación:

- Desnaturalización: 95 C por 45 segundos.

- Alineamiento: $60^{\circ} \mathrm{C}$ por 90 segundos.

- Extensión: 72ํㅡ por 90 segundos.

- 35 ciclos de amplificación:

- Desnaturalización: 95ํㅡ por 45 segundos.

- Alineamiento: $65^{\circ} \mathrm{C}$ por 90 segundos.

- Extensión: $72^{\circ} \mathrm{C}$ por 2 minutos. cación con oligonucleótidos específicos para el promotor de MGMT metilado, MGMT(M), y sin metilar, MGMT(U) (El detalle de secuencias de oligonucleótidos se observa en la Tabla 1). Los preparados utilizados fueron los siguientes:

Preparado para evaluar MGMT metila-
- 1 ciclo de extensión: 72 $\mathrm{C}$ por $4 \mathrm{mi}$ nutos.

- Mantención: 4ํㅡ.

Los productos de amplificación se visualizaron en gel de agarosa al 3,5\% en relación 3:1 con agarosa Low Melting 
Tabla 1.

Oligonucleóticos descritos para estudio de metilación mediante técnica de PCR anidado (42)

\begin{tabular}{|c|c|c|c|}
\hline & Secuencia $5^{\prime}->3^{\prime}$ & Tamaño amplicón & Referencia \\
\hline MGMT For & GGATATGTTGGGATAGTT & \multirow[t]{2}{*}{$289 \mathrm{bp}$} & \multirow[t]{2}{*}{ Palmisano et al (2000) } \\
\hline MGMT Rev & CCAAAAACCCCAAACCC & & \\
\hline MGMT(U) For & TTTGTGTTTTGATGTTTGTAGGTTTTTGT & \multirow[t]{2}{*}{$93 \mathrm{bp}$} & \multirow[t]{2}{*}{ Esteller M et al (1999) } \\
\hline MGMT(U) Rev & ААСТССАСАСТСТТССААAАACAAAACA & & \\
\hline MGMT(M) For & TTTCGACGTTCGTAGGTTTTCGC & \multirow[t]{2}{*}{$81 \mathrm{bp}$} & \multirow[t]{2}{*}{ Esteller M et al (1999) } \\
\hline MGMT(M) Rev & GCACTCTTCCGAAAACGAAACG & & \\
\hline
\end{tabular}

(NuSieve) en buffer TBE 0,5X (0,043M Tris-Base, 0,044M de ácido bórico, $1 \times 10^{-3} \mathrm{M}$ EDTA). La electroforesis se realizó a $5 \mathrm{~V} / \mathrm{cm}$ durante 90 minutos en buffer TBE 0,5X. Para corroborar el tamaño del producto de PCR se comparó con una escalera de peso molecular de 10 pares de bases (O'RangeRuler 10bp DNA Ladder, Fermentas; CAT SM1313). Para su observación, se utilizó transiluminador UV. Como referencia de controles metilados y no metilados, se electroforesis en DNA de células de cáncer de mama tipo MCF-7 como control de no metilación, y para control metilado, se realizó el proceso de metilación utilizando enzima Metiltransferasa M.Sssl (New England Biolabs CAR: M0226S) según indicaciones del fabricante en las mismas células tumorales.

\section{Análisis clínico}

Se realizó el estudio de fichas clínicas de los pacientes en cuyas muestras finalizó con éxito el estudio biomolecular. Ya que la decisión terapéutica fue determinada por el médico tratante, se consignó si ésta correspondió a biopsia, resección parcial o resección completa. Se analizó la sobrevida global en meses y se determinó el porcentaje de sobrevida al año y a los dos años, estratificándose según la presencia o ausencia de metilación del promotor de MGMT. En la construcción de la base de datos, se asignó un número según el orden de la biopsia procesada por año, evitando guardar nombre y RUT de pacientes, manteniendo el anonimato estadístico. Con respecto a la terapia complementaria postquirúrgica, al momento del presente estudio existía un protocolo de ingreso a radioquimioterapia en el Hospital Carlos Van Buren vigente desde 2009 , en el que se contaban los siguientes factores necesarios para ser candidato a radioquimioterapia:

- ECOG 0-1-2.

- Menores de 55 años.

- Mayores de 55 años con test minimental puntaje mayor o igual a 27 (medido al ingreso por neurocirujano que recibe al paciente).

- Cirugía con resección de al menos $80 \%$ de la masa tumoral evaluado con resonancia magnética postoperatoria precoz.

- Biopsia que confirme el diagnóstico de glioblastoma multiforme.

\section{Análisis estadístico}

Se realizó una base de datos utilizando Microsoft ${ }^{\circledR}$ Excel. La sobrevida global se midió a partir de la cirugía hasta el fallecimiento, estudiándose con curvas de Kaplan-Meier y evaluando las diferencias entre ellas con test de log-rank, con intervalo de confianza de $95 \%$. Para el análisis multivariado se utilizó riesgo proporcional de Cox, evaluando el valor pronóstico de la metilación del promotor de MGMT. Se consideró valores significativos con p menores a 0,05. Todos los cálculos fueron realizados utilizando el software IBM SPSS Statistics versión 20 .

\section{Financiamiento y conflictos de interés}

Este estudio conto con el apoyo financiero de Laboratorio Saval S.A., por medio de "Beca Saval de Investigación". Las bases y formularios de postulación, así como la evaluación de los proyectos fueron responsabilidad de la Dirección de la Escuela de Medicina de la Universidad de Valparaíso, a través de la Dirección de Postgrado.

\section{Resultados}

\section{Características generales}

Entre enero de 2010 y diciembre de 2014 se realizó el diagnóstico de Glioblastoma Multiforme a 55 pacientes de forma consecutiva en el Servicio de Anatomía Patológica del Hospital Carlos Van Buren. Fueron excluidos 23 pacientes, 16 pacientes por no cumplir con el criterio de inclusión de edad, seis por presentar un ECOG postoperatorio superior a 2 y uno por ser finalmente catalogado como astrocitoma anaplásico en la confirmación diagnóstica. No fue posible recuperar 5 muestras parafinadas de los archivos de Anatomía Patológica, por lo que el número muestras que se procesó fue de 28. Para corroborar la calidad del ADN, se efectuó una primera reacción de polimerasa en cadena para una región específica del exón 2 de KRAS con oligonucleótidos específicos, proceso descrito previamente, descartándose 7 muestras por mala calidad de DNA, ingresando finalmente para el estudio del estado de metilación del promotor de MGMT un total de 21 muestras de distintos pacientes.

Las características de los pacientes se detallan en la Tabla 2 . Se realizó radioterapia en 17 pacientes, recibiendo un total de 60 Gy doce pacientes y 30 Gy cinco de ellos. De los 21 pacientes que se estudiaron en el proyecto, cuatro recibieron quimioterapia con temozolomida según protocolo Stupp.

\section{Amplificación del promotor de MGMT}

De estas 21 muestras, ocho fueron obtenidas a través de biopsia estereotáxica y en 4 fue posible determinar el estado de metilación del promotor de 


\begin{tabular}{|l|c|c|}
\hline Tabla 2 & $\mathbf{n}$ & $\%$ \\
\hline Sexo & & 57,1 \\
\hline Masculino & 12 & 42,9 \\
\hline Femenino & 9 & \\
\hline Cirugía realizada & 13 & 61,9 \\
\hline Exéresis & 8 & 38,1 \\
\hline Biopsia estereotáxica & & \\
\hline Promedio de sobrevida & 12,3 meses (IC 95\%, 8,3-16,3) \\
\hline Global & 16 & 76,2 \\
\hline A los 6 meses & 9 & 42,9 \\
\hline A los 12 meses & 2 & 9,5 \\
\hline A los 24 meses & \multicolumn{2}{|c|}{} \\
\hline
\end{tabular}

MGMT, resultando en un $50 \%$ de éxito en la amplificación. Las 13 muestras restantes fueron extraídas mediante exéresis quirúrgica, pudiéndose realizar la amplificación en ocho de ellas, resultando en un $61,5 \%$ de éxito en la amplificación. Contando todas las muestras procesadas, se pudo realizar la amplificación en 12 de 21 muestras procesadas, dando como resultado general un $57,1 \%$ de éxito en la amplificación. En las Figuras 4 y 5 se muestra los resultados de electroforesis realizados.

Relación entre el tiempo de sobrevida y el tiempo de sobrevida libre de progresión con respecto al estado de metilación del promotor de MGMT

Se evaluó un total de 12 muestras en las que fue posible realizar la amplificación. El resumen de los datos obtenidos se puede observar en la Tabla 3. Destaca dentro de nuestros resultados, que en 10 de las 12 muestras se encontró un estado del promotor no metilado, correspondiente a un $83,3 \%$. Sólo en dos de las muestras se pudo observar un estado metilado del promotor $(16,7 \%)$. El promedio de sobrevida de los pacientes estudiados fue de 9,2 meses, comparado con los 21 meses de los pacientes con el promotor metilado. Comparando ambas curvas de sobrevida, apareció una diferencia importante, pero no estadísticamente significativa con respecto a la sobrevida en pacientes con promotor metilado y promotor no metilado ( $p=0,15$, Figura 6 ). Con respecto al tiempo de sobrevida libre de progresión, en el grupo no metilado el promedio fue de 6,6 meses, en contraste con los 15 meses del grupo con promotor metilado $(p=0,32)$. Cabe destacar que el número de pacientes del estudio no permite realizar resultados estadísticamente significativos, dado que el objetivo principal fue determinar la factibilidad de la realización de las técnicas biomoleculares.

\section{Discusión}

La determinación de la metilación del promotor de MGMT se ha desarrollado en distintos centros para la evaluación del pronóstico del paciente con glioblastoma multiforme, siendo más importante desde la publicación del estudio NOA-8 ${ }^{30}$. El porcentaje de población afectada por este tipo tumoral con metilación del promotor ha sido objeto de estudio en múltiples países, encontrándose un porcentaje de $47,5 \%$ en población portuguesa ${ }^{35}$, entre un $56,8 \%$ a un $62 \%$ en población india ${ }^{36,37}$, entre varios otros estudios. En una revisión realizada por Weller et al en glioblastoma, se encontraron porcentajes de pacientes con metilación de

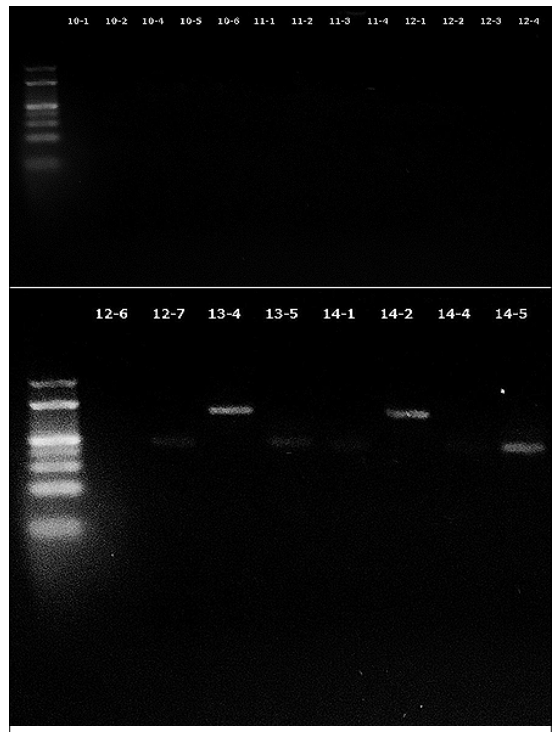

Figura 4. Electroforesis final realizada para estudio de estado de promotor metilado en muestras de glioblastoma multiforme del Hospital Carlos Van Buren entre 2010 y 2014.

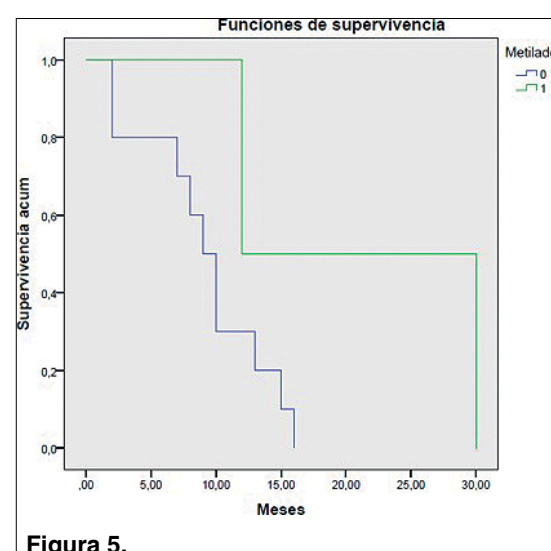

un promotor en porcentajes que varían entre un $19 \%$ a un $68 \%$, en la gran mayoría de estos estudios utilizando PCR específica para metilación basados en gel de electroforesis ${ }^{38}$. En el estudio previo realizado en nuestro centro se encontró un $68 \%$ de pacientes con promotor metilado ${ }^{33}$, distando considerablemente con lo encontrado en
Tabla 3.

Cuadro comparativo de tiempo promedio de sobrevida y de tiempo de sobrevida libre de progresión con respecto al estado de promotor de metilación

\begin{tabular}{|l|c|c|c|}
\hline & $\begin{array}{c}\text { Promotor } \\
\text { metilado }\end{array}$ & $\begin{array}{c}\text { Promotor no } \\
\text { metilado }\end{array}$ & $\mathbf{p}$ \\
\hline Sobrevida & 21 meses & 9,2 meses & 0,15 \\
\hline Sobrevida libre de progresión & 15 meses & 6,6 meses & 0,18 \\
\hline
\end{tabular}




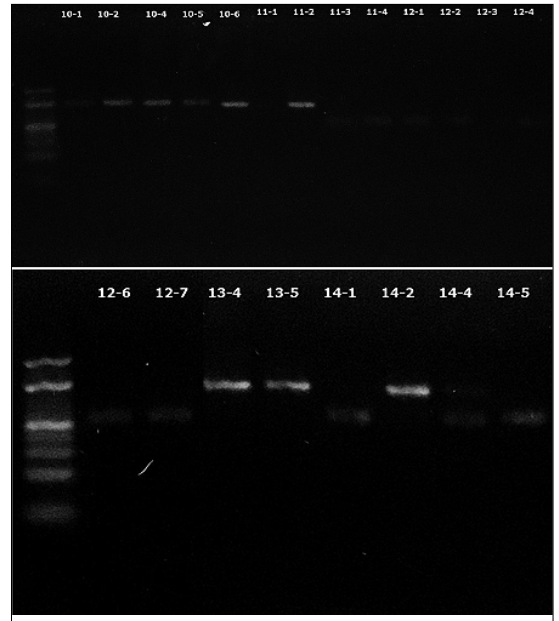

Figura 6. Electroforesis final realizada para estudio de estado de promotor no metilado en muestras de glioblastoma multiforme del Hospital Carlos Van Buren entre 2010 y 2014.

nuestro estudio, con solo un $16,7 \%$ de las muestras estudiadas con presencia del promotor metilado, siendo incluso uno de los más bajos porcentajes que pueden encontrarse actualmente en la literatura. Hay que tener en cuenta que el período de estudio fue menor y que la cantidad de pacientes también fue menor, pudiendo explicar en parte los resultados obtenidos.

La forma de determinar el estado de metilación de este promotor también ha sido objeto de revisión. En un estudio realizado por Quillien et al, se compararon cinco procesos que determinan el grado de metilación del promotor de MGMT, incluyendo PCR específica para metilación, MethyLight, metilación de alta resolución de fusión e inmunohistoquímica, evidenciándose que la mejor técnica actualmente disponible correspondería a la pirosecuenciación, en donde la PCR específica para metilación presenta una adecuada correlación con esta ${ }^{39}$. A pesar de lo anterior, en gran parte de los trabajos que evaluaron el porcentaje de metilación en glioblastoma y su relación con sobrevida y progresión utilizaron PCR específica para metilación ${ }^{38}$, técnica que fue utilizada en el presente estudio. Asimismo, el porcentaje de éxito en la amplificación descrito en la literatura varía entre un $48 \%$ hasta un $90 \%$, siendo mayor cuando se utilizan secciones crioprecipitadas con respecto a muestras parafinadas ${ }^{38,40}$. En una revisión de Hegi et al, se reporto que trabajando con muestras parafinadas se podría obtener un porcentaje de éxito en la amplificación de aproximadamente un $75 \%{ }^{41}$. En nuestro trabajo, se logro un $57,1 \%$ de éxito en la amplificación, lo que se encuentra dentro de los rangos encontrados en la literatura, aunque podría aumentarse para determinaciones futuras contando con un adecuado protocolo de conservación de muestras tumorales fijadas o introduciendo la conservación mediante crio precipitación en patologías especificas.

En nuestro estudio no se logro encontrar diferencias estadísticamente significativas en cuanto al tiempo de sobrevida y tiempo libre de progresión. La tendencia general en la literatura es que la metilación del promotor de MGMT determina una mayor sobrevida y un mayor tiempo libre de enfermedad, además de mayor efectividad frente a la radioquimioterapia posterior a la cirugía ${ }^{33,35}$. A pesar de no encontrar diferencias estadísticas, existe una tendencia a que la metilación del promotor de MGMT en nuestra población involucraría un mejor pronóstico en cuanto a tiempo de sobrevida y progresión de la enfermedad, por lo que estos resultados podrían estar influenciados por el número pequeño de pacientes estudiados. Para lograr encontrar estos resultados, se requiere ampliar el tamaño de muestra y, en lo ideal, realizar estudios prospectivos con un protocolo establecido, ya que pudimos corroborar que realizar esta técnica es factible en el Centro de Investigaciones Biomédicas de la Universidad de Valparaíso, e involucraría una evolución notoria en el estudio de patología tumoral en nuestra región.

\section{Agradecimientos}

Dr. Humberto Vallejos, anatomopatólogo, Dr. Carlo Lozano, anatomopatólogo, Camila Escobar, tecnóloga médica.

\section{Recibido: 20 de marzo de 2019 \\ Aceptado: 15 de abril de 2019}

\section{Referencias}

1. DeAngelis L. Brain Tumors. N Engl J Med 2001; 344: 114-23.

2. Schwartzbaum J, Fisher J, Aldape K, et al. Epidemiology and molecular pathology of glioma. Nat Clin Pract Neurol 2006; 2(9): 494-503.

3. Kleihues P, Cavenee W. Tumors of the central nervous system: pathology and genetics. 2a ed. Lyon: International Agency for Research on Cancer; 1997.

4. Larjavaara S, Mantyla R, Salminen T, et al. Incidence of gliomas by anatomic location. Neuro Oncol 2007; 9(3): 319-25.

5. Gousias K, Markou M, Voulgaris S, et al. Descriptive epidemiology of cerebral gliomas in northwest Greece and study of potential predisposing factors, 2005-2007. Neuroepidemiology 2009; 33(2): 89-95.

6. Louis D, Ohgaki H, Wiestler O, et al. The 2007 WHO classification of tumours of the central nervous system. Acta Neuropathol 2007; 114 : 97-109.

7. Ostrom QT, Gittleman $\mathrm{H}, \mathrm{Xu}$ J, et al. CBTRUS statistical report: primary brain and central nervous system tumors diagnosed in the United States in 2009-2013. Neuro-oncol 2016; 18(sup 5): v1-7.

8. Arora R, Alston R, Eden T, et al. Age-incidence patterns of primary CNS tumors in children, adolescents, and adults in England. Neurooncol 2009; 11(4): 403-13.

9. Lee C, Jung K, Yoo H, et al. Epidemiology of primary brain and central nervous system tumors in Korea. J Korean Neurosurg Soc 2010; 48(2): 145-52.

10. Dobes $M$, Khurana V, Shadbolt B, et al. Increasing incidence of glioblastoma multiforme and meningioma, and decreasing incidence of schwannoma (2000-2008): findings of a multicenter Australian study. Surg Neurol Int 2011; 2: 176.

11. Gigineishvili D, Shengelia N, Shalashvili G, et al. Primary brain tumour epidemiology in Georgia: first-year results of a population-based study. J Neurooncol 2013; 112(2): 241-6.

12. Louis D, Perry A, Reifenberger G, et al. The 2016 World Health Organization Classification of Tumors of the Central Nervous System: a summary. Acta Neuropathol 2016; 131(6): 803-20. 
13. Ohgaki $\mathrm{H}$, Kleihues $\mathrm{P}$. Population-based studies on incidence, survival rates, and genetic alterations in astrocytic and oligodendroglial gliomas. J Neuropathol Exp Neurol 2005; 64(6): 479-89.

14. Fisher J, Schwartzbaum J, Wrensch M, et al. Epidemiology of brain tumors. Neurol Clin 2007; 25(4): 97-109.

15. Farrell C, Plotkin S. Genetic causes of brain tumors: neurofibromatosis, tuberous sclerosis, von Hippel-Lindau, and other syndromes. Neurol Clin 2007; 25(4): 925-46.

16. Chang S, Parney I, Huang W, et al. Glioma Outcomes Project Investigators. Patterns of care of adults with newly diagnosed malignant glioma. JAMA 2005; 293(5): 557-64.

17. Stupp R, Mason W, van der Bent M, et al. Radiotherapy plus concomitant and adjuvant temozolomide for glioblastoma. N Engl J Med 2005; 352: 987-96.

18. Lacroix M, Abi-Said D, Fourney D, et al. A multivariate analysis of 416 patients with glioblastoma multiforme: prognosis, extent of resection, and survival. J Neurosurg 2001; 95(2): 190- 98.

19. Walker M, Alexander $\mathrm{E} \mathrm{Jr}$, Hunt W, et al. Evaluation of BCNU and/or radiotherapy in the treatment of anaplastic gliomas: a cooperative clinical trial. J Neurosurg 1978; 49(3): 333-43.

20. Claes A, Idema AJ, Wesseling P. Diffuse glioma growth: a guerrilla war. Acta Neuropathol 2007; 114(5): 443-58

21. Barker F 2nd, Prados M, Chang S, et al. Radiation response and survival time in patients with glioblastoma multiforme. J Neurosurg 1996; 84(3): 442-8.

22. Butowski N, Lamborn $\mathrm{K}$, Berger M, et al. Historical controls for phase II surgically based trials requiring gross total resection of glioblastoma multiforme. J Nuerooncol 2007; 85(1): 87-94.

23. Keles G, Chang E, Lamborn K, et al. Volumetric extent of resection and residual contrast enhancement of initial surgery as predictors of outcome in adult patients with hemispheric anaplastic astrocytoma. J Neurosurg 2006; 105(1): 34-40.

24. Hwang B, Chaichana K, Quiñones-Hinojosa A. The role of surgery in the management of High-grade gliomas (Newly diagnosed, recurrent, and multifocal high-grade gliomas). In: Quiñones-Hinojosa A, Raza S, editors. Controversies in Neuro-Oncology. New York: Thieme; 2014.

25. Laperriere N, Zuraw L, Cairncross G. Cancer Care Ontario Practice Guidelines Initiative Neuro-Oncology Disease Site Group. Radiotherapy for newly diagnosed malignant glioma in adults: a systematic review. Radiother Oncol 2002; 64(3): 259-73.

26. Ostrom Q, Bauchet L, Davis F, et al. The epidemiology of glioma in adults: a "state of the science" review. Neuro-oncol 2014; 16(7): 896913.

27. Agarwala S, Kirkwood J. Temozolomide, a novel alkylating agent with activity in the central nervous system, may improve the treatment of advanced metastatic melanoma. Oncologist 2000; 5: 144-51.

28. Hegi M, Diserens A, Godard S, et al. Clinical trial substantiates the predictive value of O-6-methylguanine-DNA methyltransferase promoter methylation in glioblastoma patients treated with temozolomide. Clin Cancer Res 2004; 10(6): 1871-4.

29. Esteller M. CpG island hypermethylation and tumor suppressor genes: a booming present, a brighter future. Oncogene 2002; 21: 542740.

30. Wick $\mathrm{W}$, Platten $\mathrm{M}$, Meisner $\mathrm{C}$, et al. Temozolomide chemotherapy alone versus radiotherapy alone for malignant astrocytoma in the elderly: the NOA-08 randomised, phase 3 trial. Lancet Oncol 2012; 13: 707-15.

31. Malmström A, Grønberg B, Marosi C, et al. Temozolomide versus standard 6-week radiotherapy versus hypofractionated radiotherapy in patients older than 60 years with glioblastoma: The Nordic randomised, phase 3 trial. Lancet Oncol 2012; 13: 916-26.

32. Stupp R, Hegi M, Mason W, et al. Effects of radiotherapy with concomitant and adjuvant temozolomida versus radiotherapy alone on survival in glioblastoma in a randomised phase III study: 5-year analysis of the EORTC-NCIC trial. Lancet Oncol 2009; 10: 459-66.

33. Rey González F. "Determinación del estado de metilación del promotor de MGMT por PCR anidado en muestras de glioblastoma multiforme". Tesis de especialidad médica de Radioterapia Oncológica. Profesor Guía: José Solís Campos. Biblioteca Universidad de Valparaíso, Escuela de Medicina, 2011.

34. Lorenzoni J, Torrico A, Villanueva $\mathrm{P}$, et al. Surgery for high-grade gliomas in a developing country: survival estimation using a simple stratification system. Surg Neurol 2008; 70(6): 591-97.

35. Costa B, Caeiro C, Guimarães I, et al. Prognostic value of MGMT promoter methylation in glioblastoma patients treated with temozolomide-based chemoradiation: A Portuguese multicentre study. Oncol Rep 2010; 23: 1655-62.

36. Nehru G, Pai R, Samuel P, et al. Status of O 6 - methylguanine-DNA methyltransferase [MGMT] gene promoter methylation among patients with glioblastomas from India.Neurol India 2012; 60: 481- 486.

37. Jha P, Suri V, Jain A, et al. O 6 - methylguanine DNA methyltransferase gene promoter methylation status in gliomas and its correlation with other molecular alterations: First Indian report with review of challenges for use in customized treatment. Neurosurgery 2010; 67: 1681-91.

38. Weller M, Stupp R, Reifenberger G, et al. MGMT promoter methylation in malignant gliomas: ready for personalized medicine? Nat Rev Neurol 2010; 6: 39-51.

39. Quillien V, Lavenu A, Karayan-Tapon L, et al. Comparative assessment of 5 methods (methylation- specific polymerase chain reaction, MethyLight, pyrosecuencing, methylation-sensitive high-resolution melting, and immunohistochemistry) to analyze O6-methylguanineDNA-methyltransferase in a series of 100 glioblastoma patients. Cancer 2012; 118: 4201-11.

40. Vlassenbroeck I, Califice S, Diserens A, et al. Validation of real-time MSP to determine MGMT promoter methylation in glioma. J Mol Diagn 2008; 10: 332-7.

41. Hegi M, Diserens A, Gorlia T, et al. MGMT gene silencing and benefit from temozolomide in glioblastoma. N Engl J Med 2005; 352: 9971003.

42. De la Vega Toledo E. "Estado de metilación del promotor de $\mathrm{O}^{6}$ - metilguanina-DNA Metiltransferasa en Glioblastoma Multiforme". Tesis de licenciatura en Tecnología Médica con mención en Biotecnología y Hematología. Profesor Guía: Mario Párraga San Román. Biblioteca Universidad de Valparaíso. Facultad de Medicina, 2010.

\section{Correspondencia a:}

Esteban F. Espinoza García

Salinas 1392, Casilla 150

San Felipe, Chile.

Código postal 2170000

Fono: + 56941351995

esteban.espinoza@postgrado.uv.cl 